\title{
On Robust Key Agreement Based on Public Key Authentication
}

\author{
Feng Hao \\ Thales E-Security, Cambridge, UK \\ Feng.Hao@thales-esecurity.com
}

\begin{abstract}
This paper discusses public-key authenticated key agreement protocols. First, we critically analyze several authenticated key agreement protocols and uncover various theoretical and practical flaws. In particular, we present two new attacks on the HMQV protocol, which is currently being standardized by IEEE P1363. The first attack presents a counterexample to invalidate the basic authentication in HMQV. The second attack is applicable to almost all past schemes, despite that many of them have formal security proofs. These attacks highlight the difficulty to design a crypto protocol correctly and suggest the caution one should always take.

We further point out that many of the design errors are caused by sidestepping an important engineering principle, namely "Do not assume that a message you receive has a particular form (such as $g^{r}$ for known $r$ ) unless you can check this". Constructions in the past generally resisted this principle on the grounds of efficiency: checking the knowledge of the exponent is commonly seen as too expensive. In a concrete example, we demonstrate how to effectively integrate the zero-knowledge proof primitive into the protocol design and meanwhile achieve good efficiency. Our new key agreement protocol, YAK, has comparable computational efficiency to the MQV and HMQV protocols with clear advantages on security. Among all the related techniques, our protocol appears to be the simplest so far. We believe simplicity is also an important engineering principle.
\end{abstract}

\section{INTRODUCTION}

In a seminal paper, Diffie and Hellman started the public key era by presenting a remarkably simple key agreement protocol [1]. The protocol works as follows. Suppose two users are Alice and Bob. Let $p$ be a large prime, and $\alpha$ a primitive root modulo $p$. The original scheme operates in the whole cyclic group $Z_{p}^{*}$. Alice chooses a random value $x \in_{R}[1, p-1]$ and sends $\alpha^{x}$ to Bob. Similarly, Bob chooses $y \in_{R}[1, p-1]$ and sends $\alpha^{y}$ to Alice. Finally, both parties can compute a common key $K=\alpha^{x y}$.

Later, several changes are made to the original protocol to improve security and efficiency. First, $H(K)$ is used instead of $K$ as the session key where $H$ is a one-way hash function. This is to address the issue that some (least significant) bits of $K$ may be weaker than others [2]. The second change is to move the key agreement operation from the whole group $Z_{p}^{*}$ to a large subgroup of prime order $q$ where $q \mid p-1$. This change is made to address the concern that an active attacker may confine the value $K$ to a small subgroup [24]. However, it does not really solve the problem because the protocol is unauthenticated per se. Finally, it is increasingly popular to implement the Diffie-Hellman protocol using the
Elliptic Curve Cryptography (ECC) [10]. Using ECC essentially replaces the underlying (multiplicative) cyclic group with another (additive) cyclic group defined over some elliptic curve. The essence of the protocol remains unchanged.

The acute problem with the Diffie-Hellman key agreement is that it is unauthenticated [2]. While secure against passive attackers, the protocol is inherently vulnerable to active attacks such as the man-in-the-middle attack [6]. This is a serious limitation, which for many years has been motivating researchers to find a solution [3]-[5], [7], [9], [11], [13], [20].

To add authentication, we must start with assuming some shared secret. In general, there are two approaches. The first one assumes Alice and Bob share a symmetric secret: a memorable password. Research following this line is commonly called Password Authenticated Key Exchange (PAKE) [3]-[5]. The second approach assumes Alice and Bob share some asymmetric secret: each party possesses a unique private key and his public key is known by others. In the past literature, protocols under this category are commonly called Authenticated Key Exchange (AKE) [7], [9], [11], [13], [20].

In this paper, we focus on the second category. To better differentiate it from the first category, we will call it Public Key Authenticated Key Exchange (PK-AKE). In the following section, we will review the state-of-the-art in this field.

\section{PAST WORK}

There is a large amount of literature on PK-AKE [7], [9], [11], [13], [20]. Many early protocols were constructed ad-hoc and were later found vulnerable to attacks [21]. This motivates defining a formal theoretical model to capture all attacking scenarios in PK-AKE, so that a scheme that is mathematically proved secure under the model will be immune to not only known attacks, but also undiscovered attacks [7], [11]. This is a noble aim. However, numerous attacks against the provably secure schemes suggest that defining such a model is not an easy task [13]-[15], [17].

Before reviewing past techniques in detail, we start by summarizing three general principles. These principles are important because they can help explain most of the design errors in the past.

- The sixth principle - Do not assume that a message you receive has a particular form (such as $g^{r}$ for known $r$ ) unless you can check this [22].

- The explicitness principle - Robust security is about explicitness; one must be explicit about any properties 
which can be used to attack a public key primitive, such as multiplicative homomorphism, as well as the usual security properties such as naming, typing, freshness, the starting assumptions and what one is trying to achieve [22].

- The extreme-adversary principle - Robust security is to protect against an extremely powerful adversary: the only powers that the adversary does not have are those that would allow him to trivially break any PK-AKE protocol [13].

These principles are simple and intuitive. The first two are time-honored guidance in designing robust cryptographic protocols, defined by Anderson and Needham back in 1995 [22]. The third one is a theoretical principle that we summarize from [13]. This principle is particularly important because it provides the ultimate definition of the protocol security [13]. In the following review, we will apply this extreme-adversary principle to assess the actual robustness of the protocols.

There is one big family of PK-AKE protocols based on the use of the digital signature [10]. The basic idea is to use the static private keys to digitally sign ephemeral public keys (together with some auxiliary inputs such as identities), so that man-in-the-middle attacks can be prevented. It was first described by Diffie, Oorschot and Wiener in the design of the Station-To-Station (STS) protocol [12]. Subsequent signaturebased protocols can be seen as variants of the STS protocol.

One well-known member in this family is the SIG-DH protocol due to Canetti and Krawczyk [11]. This protocol is notable for its provable security in a formal model, commonly known as the Canetti-Krawczyk (CK) model. The CK model defines a strong adversary who has the power to corrupt a session and learn all session-specific transient secrets. The goal is that a corrupted session must not impact the security of other sessions.

The SIG-DH protocol is described in Figure 1. It operates in a subgroup of $Z_{p}^{*}$ of prime order $q$. The $g$ is a generator (nonidentity element) of the subgroup. The symbols $\hat{A}, \hat{B}$ denote the user identities and $g^{a}, g^{b}$ their respective static public keys. The rest symbols are self-explanatory. More details about the SIG-DH protocol can be found in [11].

The provable security of SIG-DH is however disputed by LaMacchia et al [13]. The argument centers on the definition of the "session-specific transient secrets". In [11], the formal proofs only consider the session key and ephemeral exponents as transient secrets. The SIG-DH protocol however does not explicitly specify a digital signature scheme. In fact, for common signature schemes, such as DSA, Schnorr or ElGamal, the signing operation will introduce an additional ephemeral secret (for randomization). If that randomization secret is revealed in a corrupted session, then the static private key will be disclosed. This will surely impact on the security of other sessions, thus invalidating the claim in [11]. The same attack applies to all signature-based PK-AKE protocols. Note, this attack does not necessarily mean SIG-DH must be insecure in practice. Nonetheless, it shows the inconsistency between the claimed (or proved) security and the actual security.

To address the above deficiency, LaMacchia et al proposed an extended Canetti-Krawczyk (eCK) model [13]. The new model assumes the attacker can learn all - instead of parts - of the session specific secrets. Accordingly, the authors presented a NAXOS protocol, and formally proved it secure under the eCK model. Their protocol is shown in Figure 2.

The eCK model claims to be the "strongest" among all formal models [13]. However, this claim is disputed by Cremers [15]. He compares the theoretical properties between the the $\mathrm{CK}$ and $\mathrm{eCK}$ models, and demonstrates that a protocol proven secure in the eCK model may prove insecure in the CK model. In other words, the two models are simply incompatible: neither one is stronger than the other (also see [28]).

The problem in LaMacchia et al's model is that the definition of "session specific secrets" is still ambiguous. Notice in Figure 2, Alice uses $H_{1}(x, a)$ instead of $x$ on the exponent a technique known as the "NAXOS trick" [28]. Similarly, Bob uses $H_{1}(y, b)$ instead of $y$. The underlying assumption in the NAXOS formal proofs is that the attacker has to steal both the ephemeral secret $x$ and the static private key $a$ in order to learn the exponent. This assumption plays a vital role in proving security in the eCK model. However, one will naturally ask whether $H_{1}(x, a)$ itself forms part of the "session specific secrets". Allowing a powerful attacker to learn one transient secret $x$ but denying him to learn another transient secret $H_{1}(x, a)$ contradicts the extreme-adversary principle stated in the NAXOS paper [13].

There is a secondary reason for using $H_{1}(x, a)$ instead of $x$ in NAXOX [13]. That is to address the problem that "the random number generator of a party is corrupted". In that case, the ephemeral secret $x$ will have low entropy. Consequently, an attacker may be able to uncover $x$ say by exhaustive search. On the other hand, if the low-entropy $x$ is combined with a high-entropy private key $a$ to form $H_{1}(x, a)$, the exponent will have high entropy. As plausible as this analysis may sound, it fails to consider the correlation between the exponents. Figure 3 shows a replay attack if the random number generator is corrupted. Assume in one past session, Alice had transferred $\$ 1 \mathrm{~m}$ to Charlie. Since $x$ has low entropy, with some nonnegligible probability the same $x$ value may repeat in a future session. When that occurs, the attacker simply replays the old values $Y$ and $M$ as in the past session, to cause Alice to transfer money again.

This attack shows that hashing $x$ together with $a$ does not really solve any problem. Even worse, it may provide a false sense of security. In fact, if the end user's random number generator is corrupted, no PK-AKE protocols can guarantee security under that setting. The NAXOS protocol is of course no exception.

We now move on to study a different protocol: HMQV (see Figure 4) [7]. The HMQV protocol is modified from MQV [20] with the primary aim for provable security. The modifications come in two favors. First, HMQV uses a hash function to derive $d$ and $e$ instead of a linear function as defined in MQV. It also mandates the use of a hash function to derive the session key. Second, HMQV provably drops some mandated verification steps in MQV, including the Proof of Possession check during the CA registration and the primeorder validation check of the ephemeral public key.

The changes in the second category are highly controversial 


\begin{tabular}{|lccc|}
\hline & Alice $\left(\hat{A}, g^{a}\right)$ & Bob $\left(\hat{B}, g^{b}\right)$ \\
\hline 1. & $x \in_{R} Z_{q}$ & $\hat{A}$, sid, $g^{x}$ & \\
2. & Verify signature & $\frac{\hat{B}, \text { sid, } g^{y}, S I G_{B}\left(\hat{B}, \text { sid }, g^{y}, g^{x}, \hat{A}\right)}{\stackrel{\hat{A}, \text { sid, } S I G_{A}\left(\hat{A}, \text { sid }, g^{x}, g^{y}, \hat{B}\right)}{\longrightarrow}}$ & Verify signature \\
3. & & Alice and Bob compute $\kappa=H\left(g^{x y}\right)$ & \\
\hline
\end{tabular}

Figure 1. SIG-DH protocol. The session identifier sid is unique among all sessions owned by $\hat{A}$, the initiator.

\begin{tabular}{|c|c|c|}
\hline Alice $\left(\hat{A}, g^{a}\right)$ & & $\operatorname{Bob}\left(\hat{B}, g^{b}\right)$ \\
\hline$x \in_{R} Z_{q}$ & $X=g^{H_{1}(x, a)}$ & Verify $X$ has prime order $q$ \\
\hline Verify $Y$ has prime order $q$ & $Y=g^{H_{1}(y, b)}$ & $y \in_{R} Z_{q}$ \\
\hline
\end{tabular}

Figure 2. NAXOS protocol. The $H_{1}$ and $H_{2}$ are two independent hash functions.

\begin{tabular}{|c|c|c|c|}
\hline \multicolumn{3}{|c|}{ Alice $\left(\hat{A}, g^{a}\right)$} & Attacker (pretend "Bob") \\
\hline 1. & $x$ (repeat) & $X=g^{H_{1}(x, a)}$ & Detect same $X$ as in the past \\
\hline \multirow[t]{3}{*}{2 . } & Verify $Y$ has prime order $q$ & $\overrightarrow{Y=g^{H_{1}(y, b)}}$ & Replay old $Y$ \\
\hline & Pay Charlie $\$ 1 \mathrm{~m}$ & $M=E_{\kappa}($ "Transfer $\$ 1 \mathrm{~m}$ to Charlie" $)$ & Replay old $M$ \\
\hline & Alice comput & $\kappa=H_{2}\left(g^{a \cdot H_{1}(y, b)}, g^{b \cdot H_{1}(x, a)}, g^{H_{1}(y, b) \cdot H}\right.$ & $(x, a), \hat{A}, \hat{B})$ \\
\hline
\end{tabular}

Figure 3. Replay attack on NAXOS protocol if $x$ has low entropy

\begin{tabular}{|c|c|c|c|}
\hline & Alice $\left(\hat{A}, g^{a}\right)$ & & $\operatorname{Bob}\left(\hat{B}, g^{b}\right)$ \\
\hline 1. & $x \in_{R} Z_{q}$ & $X=g^{x}$ & Verify $X \neq 0$ \\
\hline 2. & Verify $Y \neq 0$ & $Y=g^{y}$ & $y \in_{R} Z_{q}$ \\
\hline
\end{tabular}

Alice computes: $\kappa=H\left(\left(Y B^{e}\right)^{x+d a}\right)=H\left(g^{(x+d a)(y+e b)}\right)$

Bob computes: $\kappa=H\left(\left(X A^{d}\right)^{y+e b}\right)=H\left(g^{(x+d a)(y+e b)}\right)$

Figure 4. HMQV protocol. $\bar{H}$ and $H$ are two independent hash functions.

despite that they are backed up by a formal model and full proofs [7]. Dropping the public key validations is the direct cause of several attacks against HMQV [14], [17]. In one example, Menezes and Ustaoglu demonstrated a small subgroup confinement attack that could lead to the disclosure of the user's private key [14]. That attack assumes a corrupted session where the attacker can learn the ephemeral exponent. This assumption is allowed in the original adversarial model in HMQV, hence the attack is valid. In the subsequent submission to IEEE P1363 Working Group [8], Krawczyk revised the HMQV protocol by adding the following $\operatorname{check}^{1}$ : Alice verifies the term $Y B^{e}$ has the correct prime order and Bob does the same for $X A^{d}$. This change prevents the attack reported in [14], but decreases the claimed efficiency of HMQV. The revised HMQV had been included into the IEEE P1363 standards draft (2009-06-30) [29].

\footnotetext{
${ }^{1}$ Actually, Krawczyk does not mandate this check in [8]. He specifies that such a check is necessary to thwart a strong adversary and not necessary if the adversary is less powerful. This is ambiguous. In this paper, we only analyze the stronger (or more secure) version of the revised HMQV, in which the additional check is in place.
}

However, the revised HMQV still has flaws. First, we present a new "invalid public key attack" that exploits the lax $\mathrm{CA}$ requirement in HMQV. In both the original and revised versions of $\mathrm{HMQV}, \mathrm{CA}$ is only required to check the submitted public key is not 0 . The attack works as follows. Assume Bob (attacker) registers a small group element $s \in G_{w}$ as the public key where $w \mid p-1$. Bob chooses an arbitrary value $z \in Z_{q}$. Let $Y=g^{z} \cdot s^{\prime}$ where $s^{\prime}$ is an element in the same small subgroup $G_{w}$. Exhaustively, Bob tries every element $s^{\prime}$ in $G_{w}$ such that $Y B^{e}=g^{z} \cdot s^{\prime} \cdot s^{e}=g^{z}$. In other words, the small subgroup elements $s$ and $s^{\prime}$ cancel each other out. Suppose $\bar{H}$ works like a random oracle as assumed in HMQV (see Figure 4). Then, for each try of $s^{\prime}$, the probability of finding $s^{\prime} \cdot s^{e}=1$ is $1 / w$. It will be almost certain to find such $s^{\prime}$ after searching all $w$ elements in $G_{w}$ (if not then change a different $z$ and repeat the procedure). Following the HMQV protocol, Bob sends $Y=g^{z} \cdot s^{\prime}$ to Alice. Alice checks $Y B^{e}$ has the correct prime order and computes the session key $\kappa=H\left(\left(Y B^{e}\right)^{x+d a}\right)=H\left(g^{z \cdot(x+d a)}\right)$. Because Bob knows $z$, he can compute the same session key $\kappa$ and successfully authenticates himself to Alice. In fact, anyone can do the same pre-computation as above and authenticate to Alice as "Bob".

The fact that an obviously invalid public key is totally undetected by all flows in HMQV is unsettling. For any PKAKE protocol, the basic goal of authentication is to assure one party that the other party is the legitimate holder of the supplied public key certificate - more technically, someone who knows the private key [2]. However, in this case, the private key does not even exist, but the authentication is successful. This indicates a protocol design error. Among a number of key agreement schemes [9], [11], [13], [20], it 


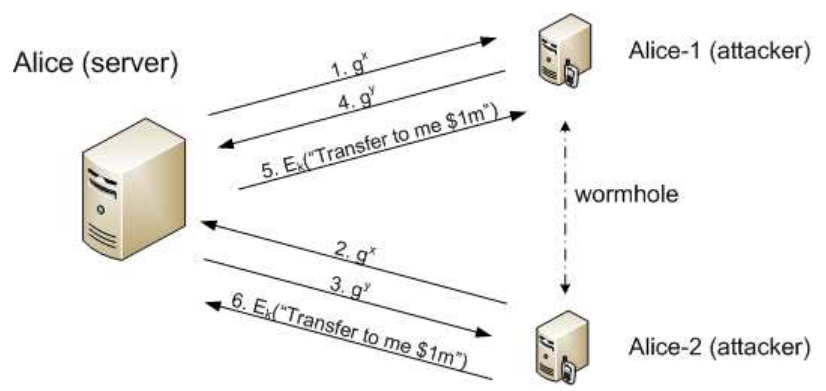

Figure 5. Worm-hole attack on HMQV

seems only HMQV has this problem. (The NAXOS protocol has the same kind of lax CA requirement as HMQV, but it mandates the end user to validate the certified public keys before any key exchange. [13])

We now describe a different "wormhole attack" on HMQV. This attack works when the two parties use the same certificate for self-communication. Self-communication is considered a useful application in [7]. For example, a mobile user and the desktop computer may hold the same static private key (registering two public key certificates costs more). Krawczyk formally proved that self-communication is "secure" in HMQV [7]. However, the formal model in [7] only considers the user talking to one copy of self, but neglects the possibility that the user may talk to multiple copies of self at the same time.

The following attack works similar to a typical "wormhole attack" in wireless networks where the attacker replicates the identity from one place to another through a wormhole tunnel [6]. Figure 5 illustrates the steps of the attack:

1) Alice initiates the connection to a copy of herself by sending $g^{x}$. The connection is intercepted by Mallory who pretends to be Alice-1.

2) Mallory starts a separate session by pretending to be Alice-2. He initiates the connection by sending to Alice $g^{x}$ (this is possible because HMQV does not require the sender to know the exponent).

3) Alice responds to Alice-2 by sending $g^{y}$.

4) Mallory replays $g^{y}$ to Alice as Alice- 1 .

5) Alice derives a session key and sends an encrypted message to Alice-1, say: "Transfer to me $\$ 1 \mathrm{~m}$ ".

6) Mallory replays the encrypted message to Alice. (After receiving money from Alice, Mallory disconnects both connections.)

In the above attack, we only demonstrated the attack against the two-pass HMQV (implicit authentication). For the threepass HMQV (explicit authentication), the attack works exactly the same. Also, we have omitted the identities in the message flows, because they are all identical (see [7]).

This attack is essentially an unknown key sharing attack. Alice thinks she is communicating to a mobile user with the same certificate, but she is actually communicating to herself. The attacker does not hold the private key, but he manages to establish two fully authenticated channels with Alice (server). In a different attacking scenario, if Alice sends an encrypted command "shutdown" to its mobile user in step 5, the same command may be replayed back to Alice to shutdown the system. This shows such an attack can be dangerous. The same attack also applies to other PK-AKE schemes, including NAXOS [13], KEA+ [9], CMQV [18], MQV [20], and SIGDH [11] etc.

\section{THE YAK PROTOCOL}

In this section, we explore a new approach to construct the PK-AKE protocol. So far, almost all of the past PKAKE protocols [7], [9], [11], [13], [18] have sidestepped the sixth robustness principle that we explained in Section II. The reason has mainly been for the concern on efficiency: verifying the knowledge on the exponent is considered too expensive [7], [11]. In the following sections, we will demonstrate how to effectively integrate the zero-knowledge primitive into the protocol design and meanwhile achieve good efficiency.

Our new PK-AKE protocol is called $\mathrm{YAK}^{2}$. For simplicity, we describe it in the DSA-like cyclic group setting [2], [10] (the protocol works basically the same in the ECDSA-like setting where an additive cyclic group over some elliptic curve is used). Let $G$ denote a subgroup of $Z_{p}^{*}$ with prime order $q$ in which the Computational Diffie-Hellman problem (CDH) is intractable. Let $g$ be a generator in $G$ (any non-identity element in $G$ can be used as a generator). The two communicating parties, Alice and Bob, both agree on $(G, g)$.

\section{A. stage 1: public key registration}

In stage 1, Alice and Bob exchange an authentic copy of each other's static public key. There are two ways to do this. A naive approach is that Alice and Bob meet in person. Alice selects a random secret $a \in_{R} Z_{q}$ as her private key. Similarly, Bob selects $b \in_{R} Z_{q}$ as his private key.

Personal Registration: Alice gives Bob $g^{a}$ with a knowledge proof for $a$. Similarly, Bob gives Alice $g^{b}$ with a knowledge proof for $b$.

Alternatively, this can be done via a trusted third party: Certificate Authority (CA) in a PKI.

CA Registration: Alice sends to the CA $g^{a}$ with a knowledge proof for $a$. Similarly, Bob sends to the CA $g^{b}$ with a knowledge proof for $b$.

We provide two registration methods to help explain security. The two methods are actually equivalent though they look quite different. In the first approach, Alice and Bob act like a personal " $\mathrm{CA}$ " for each other. Alice verifies Bob's identity and checks the knowledge proof to ensure Bob possesses the private key. Bob does the same. In the second approach, the CA resumes the responsibility to verify the applicant's identity (Distinguished Name) and check the knowledge proof to ensure the Proof of Possession (PoP) of the private key. The PoP check is a mandatory requirement for the CA, as stated in all PKI standards (see [19]). Because of the involvement of a trusted third party, the second approach is more scalable than the first, but it requires users to trust the CA.

\footnotetext{
${ }^{2}$ The yak lives in the Tibetan Plateau where environmental conditions are extremely adverse.
} 
In both approaches, the sender needs to produce a valid knowledge proof to demonstrate the Proof of Possession (PoP) of the private key. Fortunately, Zero Knowledge Proof is a well-established primitive in cryptography [10]. It allows the sender to prove the knowledge of the exponent without leaking it. For example, we can use Schnorr's signature, which is made non-interactive by applying the Fiat-Shamir heuristics to an interactive Schnorr identification protocol [27]. Let $H$ be a secure hash function. This function works like a random oracle (replacing the honest verifier who supplies random challenges in the interactive identification protocol). To prove the knowledge of the exponent for $X=g^{x}$, one sends \{SignerID, OtherInfo, $\left.V=g^{v}, r=v-x \cdot h\right\}$ where SignerID is the unique user identifier (also called Distinguished Name [2]), OtherInfo includes auxiliary information to indicate this is a request for certifying a static public key and may include other practical information such as the name of the algorithm etc, $v \in_{R} \quad Z_{q}$ and $h=H(g, V, X$, SignerID, OtherInfo). The receiver checks that $X$ has prime order $q$ and verifies that $V=g^{r} X^{h}$ (computing $g^{r} X^{h}$ requires roughly one exponentiation using the simultaneous computation technique [10]). We will assess the cost in more detail in Section VI.

In the past literature, several papers allow arbitrary key registration at the $\mathrm{CA}$ [7], [9], [13]. In other words, the CA indiscriminately certifies any binary string even if it is obviously not a valid public key (say a small subgroup element). For example, the NAXOS protocol only requires the CA to check the public key is not 0 [13]. But, it mandates that the users must verify the order of each other's certified public keys before the key agreement. The cost of this expensive operation is however not counted in the NAXOS paper.

To have a meaningful discussion, we need to assume a properly functional CA (the same assumption is made in [20]). As stated in every PKI standard, a CA must verify the PoP before certifying the public key [19]. It must also check the user's identity properly. If the CA is not trustworthy in fulfilling its duties, then we may have to revert to the personal registration approach.

\section{B. stage 2: key agreement}

Alice and Bob execute the following protocol to establish a session key. For simplicity of discussion, we explain the case that Alice and Bob have different certificates $(a \neq b)$ and will cover self-communication later.

YAK protocol: Alice selects $x \in_{R} Z_{q}$ and sends out $g^{x}$ with a knowledge proof for $x$. Similarly, Bob selects $y \in Z_{q}$ and sends out $g^{y}$ with a knowledge proof for $y$.

When this round finishes, Alice and Bob verify the received knowledge proof to ensure the other party possesses the ephemeral private key. As explained earlier, we can use Schnorr's signature to realize the knowledge proof. Both parties also need to ensure the identity (i.e., SignerID) in the knowledge proof must match ${ }^{3}$ the one in the public key

\footnotetext{
${ }^{3}$ By "match", we mean the identity is identical to the one on the X.509 certificate, or the two have an unambiguous one-to-one mapping relationship. The latter is useful to provide anonymity in key agreement: both parties use pseudo-identities to prove the possession of the ephemeral exponents and they know how to match the pseudo-identities to real ones at the two ends.
}

certificate.

Upon successful verification, Alice computes a session key $\kappa=H\left(\left(g^{y} \cdot g^{b}\right)^{x+a}\right)=H\left(g^{(x+a)(y+b)}\right)$. And Bob computes the same key: $\kappa=H\left(\left(g^{x} \cdot g^{a}\right)^{y+b}\right)=H\left(g^{(x+a)(y+b)}\right)$. The protocol has the same round efficiency and symmetric property as the original Diffie-Hellman protocol [1]. Figure 6 shows how to implement the protocol in two passes, as one party usually needs to initiate the connection.

The two-pass YAK protocol can serve as a drop-in replacement for face-to-face key exchange. It is equivalent to Alice and Bob meeting in person and secretly agreeing a common session key. After Alice and Bob depart, they can use the session key to secure the communication. So far, the authentication is implicit: Alice believes only Bob has the same key and vice versa. In some applications, Alice and Bob may want to perform an explicit key confirmation before starting any communication just to make sure the other party actually holds the same session key.

The method for explicit key confirmation is generally applicable to all key exchange protocols. Often, it is considered desirable to use a different key from the session key $\kappa$ for key confirmation ${ }^{4}$, say use $\kappa^{\prime}=H(K, 1)$. We summarize a few methods here. A simple method is to use a hash function as presented in [3]: Alice sends $H\left(H\left(\kappa^{\prime}\right)\right)$ to Bob and Bob replies with $H\left(\kappa^{\prime}\right)$. Another straightforward way is to use $\kappa^{\prime}$ to encrypt a known value (or random challenge) as explained in [2]. Other approaches make use of MAC functions as suggested in [7], [9]. Given that the underlying functions are secure, these methods do not differ significantly in security.

\section{SECURITY ANALYSIS}

A common approach in past work is to model an adversary in terms of what he is capable of. This methodology evolves progressively over the past decade by adding more power to the attacker. In this section, we attempt a new approach. Instead of defining what the attacker is capable of, we focus on what the attacker is not capable of. As we will demonstrate, this allows capturing the crux of the extreme-adversary principle more directly.

First, we need to define what are the "session specific secrets" in YAK. Simply put, they include all transient secrets in a session. More specifically, the session specific secrets for Alice - include the ephemeral exponent $x$ and the raw session key $K$. This definition has covered the randomization factor $v$ in Schnorr's signature since one can easily compute $v$ from $x$ and the public items within Schnorr's signature. It has also covered the session key $\kappa$, which can be computed from $H(K)$. If the attacker is powerful enough to access Alice's session state, we assume he can learn all of the transient secrets including $x$ and $K$.

So, a powerful attacker is able to learn all transient secrets in a session, but he cannot learn the user's private key. This is consistent with reality. In the real world, the standard practice is to store the private key in a Hardware Security Module

\footnotetext{
${ }^{4}$ Using a different key for key confirmation has a (subtle) theoretical advantage that after the key confirmation, the session key is still indistinguishable from random. However, this trick has limited pratical signifiicance and is not used in for example [3], [4].
} 


\begin{tabular}{|lclc|}
\hline & Alice $\left(\hat{A}, g^{a}\right)$ & & Bob $\left(\hat{B}, g^{b}\right)$ \\
\hline 1. & $x \in_{R} Z_{q}$ & $\stackrel{g^{x}, K P\{x\}}{g^{y}, K P\{y\}}$ & Verify $K P\{x\}$ \\
2. & Verify $K P\{y\}$ & $y \in_{R} Z_{q}$ \\
& Compute $\kappa=H\left(g^{(x+a)(y+b)}\right)$ & & $\kappa=H\left(g^{(x+a)(y+b)}\right)$ \\
\hline
\end{tabular}

Figure 6. YAK protocol

(HSM) [6]. The HSM may consist of two security boundaries [15]. The inner boundary has an embedded processor and limited memory. It is heavily protected and is where the private key is stored. Due to the extremely constrained resource, the embedded processor only performs the most critical operation: raising the base to the power of the private key. Thus, inner boundary works like a private key oracle, whose access must be denied to attackers. The outer boundary has more abundant computing resources. It performs all the remaining operations. However, the outer boundary is relatively less protected. So, it might leak transient information (say through side-channels [6] to an attacker nearby). Hence, the goal of a robust key agreement protocol is to minimize the trust on both the inner and outer boundaries as much as possible.

First, we formulate the following requirements for the PKAKE protocol.

1) Private key security: An attacker cannot learn any useful information about the user's static private key even if he is able to learn all session specific secrets in any session.

2) Full forward secrecy: Session keys that were securely established in the past uncorrupted sessions will remain secure in the future even when both users' static private keys are disclosed.

3) Session key security: An attacker cannot compute the session key if he impersonates a user but has no access to the user's private key.

These requirements summarize essential security properties of a PK-AKE protocol. They even cover those that are missing in the existing formal model definitions. The first requirement is generally not covered by a formal model, but we think it is crucially important. For example, both the SIG-DH [11] and (original) HMQV [7] protocols have been formally proven secure in the CK model. Yet attacks reported in [13] and [14] show that in both protocols, an attacker is able to disclose the user's private key. In the second requirement ${ }^{5}$, we use "full" to distinguish it from the "half" forward secrecy, which only allows one user's private key to be revealed (e.g., KEA+ [9]). In the past literature it is common to add "perfect" before "forward secrecy" [7], [9], [11]. However, we drop "perfect" here because it has no concrete meaning [10], [20]. The third requirement concerns both the secrecy and authenticity of the session key. It has already covered the Key Compromise Impersonation (KCI) attack [20]. The "invalid public key" attack in Section II indicates that HMQV does not satisfy this property.

\footnotetext{
${ }^{5}$ It is essentially the same as the weak Perfect Forward Secrecy (wPFS) defined in [7].
}

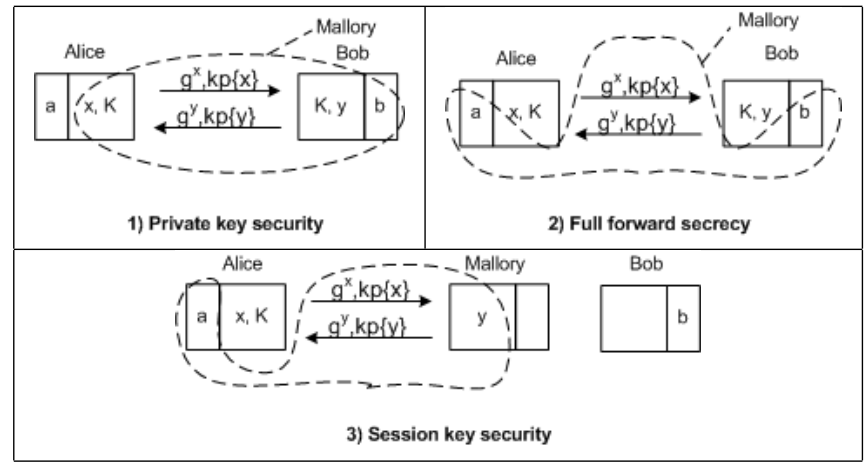

Figure 7. The oracle diagrams for the attacker. Alice is honest.

The strategy of our design is to make the best use of wellestablished techniques such as Schnorr's signature. This allows us to leverage upon the provable results of Schnorr's signature (see [10], [27]), and thus greatly simplify the security analysis. In the following, we will aim to provide a simple and intuitive security analysis.

First, Let us discuss the private key security. Without loss of generality we assume Alice is honest. Unless mentioned otherwise, this assumption will be made throughout the rest of the analysis. As shown in Figure 7 (1), Mallory totally controls Bob's static and ephemeral private keys; additionally, he has the extreme power that allows him to learn Alice's transient secrets in an arbitrary session. The only power that he does not have is the access to Alice's private key.

Claim 1 (Private Key Security): An attacker can not learn any useful information about Alice's static private key even if he is able to learn all transient secrets in any of Alice's sessions.

proof. As shown in Figure 7 (1), an extremely powerful attacker completely corruptes Bob and has access to all of the transient secrets in Alice's session. The knowledge proofs ${ }^{6}$ defined in the YAK protocol prove that the attacker knows the values of $y$ and $b$ (i.e., these variables are not correlated with a). He also knows Alice's public key $g^{a}$. By revealing Alice's transient secrets in a session, he learns $x$ and the raw session key $K=g^{(a+x)(b+y)}$. But learning $K$ does not give Mallory any infomation, because he can compute it by himself from $\left\{x, y, b, g^{a}\right\}$. Since Mallory knows the values of $\left\{x, y, b, g^{a}\right\}$, he can effectively simulate the same session all by himself by defining arbitrary values of $x, y, b$. Clearly, he does not learn any useful information about Alice's private key from his own simulations.

${ }^{6}$ If Schnorr's signature is used to realize the knowledge proof, we need to add a random oracle assumption (i.e., a secure one-way hash function), as Schnorr's signature is provably secure in the random oracle. 
Intuitively, the above proof assumes an attacker simulating a (gigantic) list of transcripts that include arbitrary values of $\{x, y, b\}$. By corrupting any of Alice's sessions, the attacker learns nothing more than what he can possibly simulate.

On the other hand, the same simulation does not work in NAXOS and HMQV. Take NAXOS as an example. Assume Bob (the attacker) sends to Alice $g^{a}$. By accessing Alice's transient items within the key derivation function, Bob learns $g^{a \cdot a}$. Bob cannot simulate the session because he cannot compute $g^{a \cdot a}$ by himself. In another session, Bob can send to Alice $g^{a^{2}}$ and then learn $g^{a^{3}}$. Similarly, he can learn $g^{a^{4}}, g^{a^{5}} \ldots$. In other words, every corrupted session gives the attacker new information that he cannot learn by simulation. The same argument applies to HMQV.

The use of the knowledge proofs greatly simplifies the analysis. Without the knowledge proofs, the simulation in our proof will not work. We illustrate this with an example. Assume there were no knowledge proof (i.e., no PoP check) during the CA registration. Mallory can choose a small subgroup element, e.g., $s \in G_{w}$ where $w \mid p-1$. He then registers $s / g^{y}$ as his static public key. During the key agreement, Alice will compute $Z=\left(s / g^{y} \times g^{y}\right)^{x+a}=s^{x+a}$. If Mallory can also reveal Alice's ephemeral secret $x$, he can compute $a \bmod w$. This is the same kind of the small subgroup attack as reported against HMQV [14]. Note that in this case, the simulation in the proof no longer works: Mallory does not know the value $b$. In fact, the value $b$ does not even exist because the registered public key is not in the form of $g^{b}$ at all. This example shows the importance of the sixth robustness principle: "Do not assume the message you receive has a particular form (such as $g^{r}$ for known $r$ ) unless you can check this" [22].

Next, we discuss the full forward secrecy requirement. In the definition, we specify that the past sessions must be "uncorrupted"7, namely the session-specific transient secrets must remain unknown to the attacker. In YAK, this means $x$, $y$ and $K$ must remain unknown to the attacker. Obviously, knowing $K$ would have trivially broken the past session. Also, if Mallory can learn any ephemeral exponent $x$ or $y$ in the past session in addition to knowing both parties' static private keys (see Figure 7 (2)), he has possessed the power to trivially compromise any PK-AKE. This contradicts the extreme-adversary principle. Therefore, in the following analysis, we assume the attacker knows both Alice and Bob's private keys, but not any transient secrets in the past session.

Claim 2 (Full Forward Secrecy): Under the Computational Diffie-Hellman (CDH) assumption, an attacker who knows both parties' static private keys but not transient secrets in the past session cannot compute $K$.

proof. To obtain a contradiction, we assume the attacker can compute $K=g^{(a+x)(b+y)}$. The attacker knows the values of $a, b$ (see Figure 7 (2)). The ephemeral public keys $g^{x}$ and $g^{y}$ are public information. Therefore, he can compute $g^{a b}, g^{a y}$

\footnotetext{
${ }^{7}$ Krawczyk defines a weak Perfect Forward Secrecy (wPFS) and a strong Perfect Forward Secrecy (sPFS) [7]. We observe that both definitions are based on essentially the same assumption: the past sessions were uncorrupted. The only difference is that the latter requires explicit assurance while in the former definition the assurance is implicit. As shown in [7], any two-pass PK-AKE protocol that fulfills wPFS also trivially satisfies sPFS by adding an explicit key confirmation.
}

and $g^{b x}$. Now, we can solve the $\mathrm{CDH}$ problem as follows: given $g^{x}$ and $g^{y}$ where $x, y \in_{R} Z_{q}$, we use the attacker as an oracle to compute $g^{x y}=K /\left(g^{a b} \cdot g^{a y} \cdot g^{b x}\right)$. This, however, contradicts the $\mathrm{CDH}$ assumption.

The above proof shows that the the raw key material $K$ is incomputable to the attacker. In practice, it may not be appropriate to directly use the raw $K$ as a session key. A common approach is to apply a key derivation function such as a hash function, so the session key is $\kappa=H(K)$. The use of the hash function serves to well mix potentially weak and strong bits in the raw output and produce a session key of the desired length. This works exactly the same as in the original Diffie-Hellman protocol [1].

Finally, we study the session key security requirement. As shown in Figure 7 (3), Mallory does not hold Bob's private key but he tries to impersonate Bob. We assume the powerful Mallory even knows Alice's private key $a$. The only power he does not have is the access to Alice and Bob's session states. If Mallory can access Alice's session state, he can impersonate anyone to Alice - he just needs to "steal" the session key that Alice computes in the transient memory. Similarly, if Mallory can access Bob's session state, he can impersonate Bob to anybody by waiting until Bob computes the session key and then stealing it.

Note in this case, the assumed attacker is less powerful than the one described in Claim 1. Previously, the attacker was able to corrupt an arbitrary session of Alice's or Bob's. He however had learned no useful information than what he can simulate (see Claim 1). On discussing the session key security, we assume the attacker no longer has access to either user's session state. This change is necessary, and is consistent with the extreme-adversary principle.

Claim 3 (Session Key Security): Under the Computational Diffie-Hellman (CDH) assumption, an attacker who impersonates Bob but does not have access to Bob's static private key can not compute $K$.

proof. The attacker does not possess Bob's static private key, or have access to either Alice or Bob's session state. To obtain a contradiction, we assume Mallory is able to compute $K=g^{(a+x)(b+y)}$. Bob's public key $g^{b}$ is public information. Mallory knows Alice's private key $a$. The knowledge proof in the protocol proves that Mallory also knows the value $y$ (see Figure 7 (3)). Hence, he can compute $g^{a b}, g^{a y}$ and $g^{x y}$. Now, we can solve the $\mathrm{CDH}$ problem as follows: given $g^{b}$ and $g^{x}$ where $x, b \in_{R} Z_{q}$, we use Mallory as an oracle to compute $g^{b x}=Z /\left(g^{a b} \cdot g^{a y} \cdot g^{x y}\right)$. This, however, contradicts the CDH assumption.

Again, the knowledge proofs are essential in the above proof. We use an example to illustrate this. Let us assume there were no knowledge proof required for the ephemeral public key. Now, Mallory can send $Y^{\prime}=g^{-b}$ to Alice and successfully force the session key to be $\kappa=H(1)$. The removal of the knowledge proof gives the attacker unrestricted freedom to fabricate a message of any form. Note validating the order of $Y^{\prime}$ does not prevent the attack, because $Y^{\prime}$ has the correct prime order. Somehow, this example highlights a limitation of the prime-order validation: it only checks whether 
the message is within the designated group, but fails to check whether it is correlated with other elements in the same group. Using the knowledge proof restricts the attacker's freedom much more stringently, and defeats this attack.

\section{SElf-COMmunication}

The user identity is an important parameter in the protocol definition. In the past literature, almost all PK-AKE protocols readily use the Distinguished Name (DN) in the user's X.509 certificate as the user identity. This practice also carries over to the self-communication mode [7]. However, self-communication is a special case and should be be handled differently. In this mode, the two parties are still physically distinct entities and hence, naturally require different identities.

To enable self-communication in YAK, we need to ensure the SignerID in the Schnorr's signature remains unique. This is to prevent Bob from replaying Alice's signature back to Alice and vice versa. One solution is to simply attach an additional identifier to the mobile stations using the same certificate. For example, when Alice (server) is communicating to the $n$th copy of herself (mobile station), Alice uses "Alice" as her SignerID to generate the Schnorr's signature and the $n$th copy uses "Alice- $n$ " as its SignerID. Thus, Alice- $n$ cannot replay Alice's signature back to Alice and vice versa. This solution is also generically applicable to fix the self-communication problem in past protocols [7], [9], [11], [13], [20].

Though self-communication is considered a useful feature [7], one should be careful to enable this feature only when it is really needed. This is because, when enabled, it may have negative impact on the theoretical security. In Section IV, we have explained that, under normal operations (using different certificates), an attacker cannot learn $g^{a \cdot a}$ in any case. However, if self-communication is enabled in YAK, we essentially allow $a=b$, hence the attacker can learn $g^{a \cdot a}$ from a corrupted session. This implies we would need a stronger assumption than $\mathrm{CDH}$ to prove the "session key security". This is undesirable, but to our best knowledge, no PK-AKE protocol is reducible to the $\mathrm{CDH}$ assumption with the selfcommunication enabled. In comparison, in NAXOS [13] and HMQV [7], the attacker can learn $g^{a \cdot a}$ from a corrupted session regardless whether the self-communication is enabled (and furthermore he can learn $g^{a^{3}}, g^{a^{4}}, \ldots$ ).

\section{COMPARISON}

Finally, we compare YAK with past work in terms of security and efficiency. There are many PK-AKE protocols in the past literature. However, we can only select a few; they include SIG-DH [11], HMQV [7], MQV [20] and NAXOS [13]. These techniques are representative for a comparative analysis. MQV has be widely standardized and applied in practical applications. The rest are all well-known PK-AKE schemes with formal proofs under different formal models. Among them, HMQV is known as the "most efficient" [7] and NAXOS as the "most secure" [13]. Other PK-AKE schemes can be seen as variants of these four.

Table I summarizes the comparison results. The cost is evaluated by counting the number of exponentiations in a
DSA-like group setting or the number of multiplications in an ECDSA-like group setting. In the former case, it takes a full exponentiation to validate the prime order of a group element while in latter, this operation is essentially free. This explains the one operation difference in Table I. We briefly explain each technique below.

The SIG-DH protocol was described in [11] and formally proven secure in the Canetti-Krawczyk (CK) model. However, the paper does not explicitly specify a signature algorithm. This makes it difficult to assert the exact cost and security assumptions because they depend on the choice of the signature algorithm. The attack presented in [13] indicates SIG-DH does not fulfill the private key security requirement. Consequently, it does not satisfy the session key security requirement (since the private key security cannot be assured in the first place). Another limitation with SIG-DH is that if the user's digital signature is captured, his real identity will be revealed.

The HMQV protocol is due to Krawczyk [7]. The protocol is revised in [8] to address the Menezes-Ustaoglu's small subgroup attack by adding a prime-order validation step (otherwise, the protocol will fail the private key security requirement). This revision makes the total number of exponentiations be 3.5. The HMQV only requires the CA to check the submitted public key is not zero. However, as shown in [15], if the attacker is allowed to register " 1 " as his public key, he can launch an unknown key-sharing attack against the one-pass version of the HMQV protocol. So we add the check that the public key is not " 1 " either. We need to caution that even so, it is still not sufficient to prevent an unknown keysharing attack against the HMQV in the post model where the responder's identity is not pre-defined [17]. The "invalid public key" attack shown in Section II indicates HMQV does not fulfill the session key security requirement.

The MQV protocol was first designed by Menezes, Qu and Vanstone [20]. The original MQV design includes the user identities only in the explicit key confirmation stage. Thus, the key confirmation not only serves to confirm the equality of the session key, but also to confirm the identities of the users who are engaged in the key agreement. This arrangement has the drawback that a secure MQV would require 3 passes. As shown by Kaliski, without key confirmation, the 2-pass MQV is subject to an unknown key sharing attack [26]. In [14], Menezes revised the MQV protocol by including the user identities into the key derivation function (similar to HMQV). This change prevents the Kaliski's attack and improves the round efficiency as the 2-pass MQV can now provide implicit authentication.

The NAXOS protocol is formally proven secure in the extended Canetti-Krawczyk model (eCK) model [13]. The eCK claims to be the "strongest" formal model, but this claim is disputed in [15]. In Section II, we also pointed out a subtle flaw in the definition of the "session specific transient secrets" in the NAXOS security proofs. The NAXOS protocol requires 5 exponentiations (see Figure 2). Same as in HMQV, the protocol allows the CA to certify any non-zero binary strings as public keys. However, NAXOS requires users to verify the other party's certified public key must lie in the correct primeorder group before key agreement. This cost is however not 


\begin{tabular}{|c|c|c|c|c|c|c|c|c|c|}
\hline & $\begin{array}{c}\text { Exp } \\
\text { DL }\end{array}$ & $\begin{array}{c}\text { Mul } \\
\text { EC }\end{array}$ & $\begin{array}{c}\text { assum- } \\
\text { ptions }\end{array}$ & $\begin{array}{c}\text { CA } \\
\text { chk }\end{array}$ & $\begin{array}{c}\text { Pri-key } \\
\text { sec }\end{array}$ & $\begin{array}{c}\text { Ses-key } \\
\text { sec }\end{array}$ & FFS & $\begin{array}{c}\text { Self } \\
\text { com }\end{array}$ & $\begin{array}{c}\text { Allow } \\
\text { anonym }\end{array}$ \\
\hline SIG-DH & - & - & - & PoP & $\times$ & $\times$ & $\checkmark$ & $\times$ & $\times$ \\
\hline HMQV & 3.5 & 2.5 & GDH, RO & not 0,1 & $\checkmark$ & $\times$ & $\checkmark$ & $\times$ & $\checkmark$ \\
\hline MQV & 3.5 & 2.5 & N/A & PoP & $\checkmark$ & $\checkmark$ & $\checkmark$ & $\times$ & $\checkmark$ \\
\hline NAXOS & 5 & 4 & GDH, RO & not 0 & $\checkmark$ & $\checkmark$ & $\checkmark$ & $\times$ & $\checkmark$ \\
\hline YAK & 5 & 4 & CDH, RO & PoP & $\checkmark$ & $\checkmark$ & $\checkmark$ & $\checkmark$ & $\checkmark$ \\
\hline
\end{tabular}

Table I

COMPARISON BETWEEN PK-AKE SCHEMES.

counted in the NAXOS paper nor reflected in our table.

On the security side, which is our primary concern, the YAK protocol has clear advantages. The security of the protocol (using two different certificates) rests on the Computational Diffie-Hellman (CDH) assumption in random oracle model. In comparison, the original Diffie-Hellman protocol depends on the same $\mathrm{CDH}$ assumption. The random oracle is needed since our protocol depends on the Schnorr's signature. The formal proofs of NAXOS and HMQV depend on a less common Gap Diffie-Hellman (GDH) assumption. The GDH assumes the attacker has access to a Decision Diffie Hellman oracle but is still unable to solve the CDH problem [7], [13]. Clearly, it is a stronger assumption than $\mathrm{CDH}$.

Finally, we study the efficiency of the protocol. In YAK, Alice needs to perform the following exponentiations: one to compute an ephemeral public key (i.e., $g^{x}$ ), one to compute the knowledge proof for $x$ (i.e., $g^{v_{x}}$ ), two to verify the knowledge proof for $y$ (i.e., $Y^{q}$ and $g^{r_{y}} Y^{h_{y}}$ ) and finally one to compute the session key $(Y \cdot B)^{x+a}$. Thus, that is five in total: $\left\{g^{x}, g^{v_{x}}, Y^{q}, g^{r_{y}} Y^{h_{y}},(Y \cdot B)^{x+a}\right\}$.

Among these operations, some are merely repetitions. To explain this, let the bit length of the exponent be $L=$ $\log _{2} q$. Then, computing $g^{x}$ alone would require roughly $1.5 \mathrm{~L}$ multiplications which include $L$ square operations and $0.5 L$ multiplications of the square terms. However, the same square operations need not be repeated for other items with the common base. If we factor this in, it will take $(1+0.5 \times 3) L=2.5 L$ to compute $\left\{g^{x}, g^{v_{x}}, g^{r_{y}}\right\}$, and another $(1+0.5 \times 2) L=2 L$ to compute $\left\{Y^{q}, Y^{h_{y}}\right\}$ and finally $1.5 L$ to compute $(Y \cdot B)^{x+a}$. Hence, that is in total $6 L$, which is equivalent to $6 L / 1.5 L=4$ usual exponentiations. This is quite comparable to the 3.5 exponentiations in MQV (which cannot reuse the square terms since the bases are all different).

\section{CONCLUSION}

In this paper, we report several new attacks on the existing public-key authenticated key agreement protocols. In addition, we present a new authenticated key agreement protocol, called YAK. Our design approach is to follow time-honored engineering rules and depend on well-established cryptographic primitives such as Schnorr signature. The robustness of the protocol is analyzed under an extremely adverse condition, in which the only powers that an attacker does not have are those that would allow him to trivially break any other protocol. Overall, YAK demonstrates robust security under the Computational Diffie-Hellman assumption in the random oracle model, while achieving comparable efficiency to the "most efficient" in the past work.

\section{ACKNOWLEDGMENT}

We thank Alfred Menezes and Berkant Ustaoglu for their generous advice and invaluable comments. We thank Lihong Yang for helping improve the readability.

\section{REFERENCES}

[1] W. Diffie and M.E. Hellman, "New directions in cryptography," IEEE Transactions on Information Theory, vol. 22, pp. 644-654, 1976.

[2] D. Stinson, Cryptography: theory and practice, Third Edition, Chapman \& Hall/CRC, 2006.

[3] D. Jablon, "Strong password-only authenticated key exchange," ACM Computer Communications Review, Vol. 26, No. 5, pp. 5-26, 1996.

[4] S. Bellovin and M. Merritt, "Encrypted Key Exchange: password-based protocols secure against dictionary attacks," Proceedings of the IEEE Symposium on Research in Security and Privacy, May 1992.

[5] F. Hao, P. Ryan, "Password authenticated key exchange by juggling," the 16th International Workshop on Security Protocols, SPW'08, Cambridge, UK, May 2008

[6] R.J. Anderson, Security Engineering : A Guide to Building Dependable Distributed Systems, Second Edition, New York, Wiley 2008.

[7] H. Krawczyk, "HMQV: a high-performance secure Diffie-Hellman protocol," Advances in Cryptology - CRYPTO 2005, LNCS 3621, pp. 546566, 2005. A longer version available at http://eprint.iacr.org/2005/176. pdf.

[8] H. Krawczyk, "HMQV in IEEE P1363," submission to the IEEE P1363 Standardization Working Group, July 7, 2006. Available at http://grouper.ieee.org/groups/1363/P1363-Reaffirm/submissions/ krawczyk-hmqv-spec.pdf

[9] K. Lauter, A. Mityagin, "Security analysis of KEA authenticated key exchange protocol," PKC'06, LNCS 3958, pp. 378-394, 2006.

[10] A.J. Menezes, P.C. van Oorschot and S.A. Vanstone, Handbook of applied cryptography, CRC Press, 1996.

[11] R. Canetti, H. Krawczyk, "Analysis of key-exchange protocols and their use for building secure channels," Eurocrypt'01, pp.453-474, 2001.

[12] W. Diffie, P.C. van Oorschot, and M.J. Wiener, "Authentication and authenticated key exchanges," Designs, Codes and Cryptography, pp. 107-125, 1992.

[13] B. LaMacchia, K. Lauter, A. Mityagin, "Stronger security of authenticated key exchange," Provable Security, LNCS 4784, pp. 1-16, 2007.

[14] A. Menezes, B. Ustaoglu, "On the importance of public-key validation in the MQV and HMQV key agreement protocols," INDOCRYPT'06, LNCS 4329, pp. 133-147, 2006.

[15] C.J.F. Cremers, "Session-state reveal is stronger than ephemeral key reveal: attacking the NAXOS authenticated key exchange protocol," ACNS'09, LNCS 5536, pp. 20-33, 2009.

[16] F. Bao, R.H. Deng, H. Zhu, "Variations of Diffie-Hellman problem," Proceeding of Information and Communication Security, LNCS 2836, pp. 301-312, 2003.

[17] A. Menezes, B. Ustaoglu, "Comparing the pre- and post-specified peer models for key agreement," Information Security and Privacy, LNCS 5107, pp. 53-68, 2008.

[18] B. Ustaoglu, "Obtaining a secure and efficient key agreement protocol for (H)MQV and NAXOS," Designs, Codes and Cryptography, Vol. 46, No. 3, pp. 329-342, 2008.

[19] C. Mitchell, Security for Mobility, The Institution of Electrical Engineers, 2004.

[20] L. Law , A. Menezes , M. Qu , J. Solinas , S. Vanstone, "An efficient protocol for authenticated key agreement," Designs, Codes and Cryptography, Vol. 28 , No. 2, pp. 119-134, 2003.

[21] C. Boyd, A. Mathuria, Protocols for authentication and key establishment, Springer-Verlag, 2003. 
[22] R.J. Anderson, R. Needham, "Robustness principles for public key protocols," Crypto'95, LNCS 963, pp. 236-247, 1995.

[23] O. Goldreich, S. Micali and A. Wigderson, "How to play any mental game or a completeness theorem for protocols with honest majority," Proceedings of the nineteenth annual ACM Conference on Theory of Computing, pp. 218-229, 1987.

[24] C.H. Lim and P.J. Lee, "A key recovery attack on discrete log-based schemes using a prime order subgroup," Crypto '97, LNCS 1295, pp. 249-263, 1997.

[25] M. Bellare, R. Canetti, H. Krawczyk, "A modular approach to the design and analysis of authentication and key exchange protocols," Proceedings of the thirtieth annual ACM symposium on Theory of Computing, pp. 419-428, 1998.

[26] B. Kaliski, "An unknown key-sharing attack on the MQV key agreement protocol," ACM Transactions on Information and System Security, Vol. 4. No. 3, 2001, pp. 275-288.

[27] C.P. Schnorr, "Efficient signature generation by smart cards," Journal of Cryptology, Vol. 4, No. 3, pp. 161-174, 1991.

[28] B. Ustaoglu, "Comparing SessionStateReveal and EphemeralKeyReveal for Diffie-Hellman protocols," The Provable Security Conference, ProvSec'09, LNCS, Nov, 2009.

[29] IEEE P1363 Standard Specifications For Public-Key Cryptography, http: //grouper.ieee.org/groups/1363/index.html. 